\title{
Falls Among Wheelchair and Scooter Users with Multiple Sclerosis-A Review
}

\author{
Laura A Rice, ${ }^{1}$ Libak Abou, ${ }^{1}$ Toni Van Denend, ${ }^{2}$ Elizabeth W Peterson ${ }^{2}$ and Jacob J Sosnoff ${ }^{1,3}$ \\ 1. Department of Kinesiology and Community Health, College of Applied Health Sciences, University of Illinois at Urbana-Champaign, Champaign, IL, \\ US; 2. Department of Occupational Therapy, College of Applied Health Sciences, University of Illinois at Chicago, Chicago, IL, US; 3. Center on Health, \\ Aging and Disability, College of Applied Health Sciences, University of Illinois at Urbana-Champaign, Champaign, IL, US
}

\begin{abstract}
F alls are increasingly recognized as a threat to the safety, health and well-being of people with multiple sclerosis (MS), and emerging evidence suggests that full time wheelchair and scooter users with MS have unique fall prevention needs. This review is comprised of three parts. Part 1 summarizes findings describing influences on falls among full time wheelchair and scooter users with MS and associated clinical implications. Although further studies are needed, early findings regarding influences on fall risk operating in this specific population highlight the importance of addressing falls that occur during activities of daily living, the high frequency of falls occurring in the bathroom, and both extrinsic (e.g., wet/slippery surfaces) and intrinsic (e.g., muscle spasticity/weakness) contributors to falls. Part 2 of this review describes available evidence regarding interventions to prevent falls in full time wheelchair and scooter users with MS. Because intervention work in this area is limited to one pilot study, Part 3 presents priorities for future research and identifies the need for randomized trials evaluating fall prevention programs that address diverse fall risk factors and allow for development and attainment of individualized fall prevention goals.
\end{abstract}

\section{Keywords}

Accidental falls, multiple sclerosis,

scooter, wheelchair

Disclosures: Laura A Rice, Libak Abou, Toni Van Denend, Elizabeth W Peterson, and Jacob J Sosnoff have nothing to disclose in relation to this article.

Review Process: Double-blind peer review.

Compliance with Ethics: This article involves a review of the literature and did not involve any studies with human or animal subjects performed by any of the authors.

Authorship: The named authors meet the Internationa Committee of Medical Journal Editors (ICMJE) criteria for authorship of this manuscript, take responsibility for the integrity of the work as a whole, and have given final approval for the version to be published.

Open Access: This article is published under the Creative Commons Attribution Non-commercial License, which permits any non-commercial use, distribution, adaptation, and reproduction provided the original authors and source are given appropriate credit. (c) The Authors 2018.

Received: July 10, 2018

Accepted: September 20, 2018

Citation: US Neurology. 2018;14(2):82-7

Corresponding Author: Laura A Rice,

219 Freer Hall, 906 S. Goodwin Ave. Urbana,

IL 61801, US. E: ricela@illinois.edu

Support: No funding was received in

the publication of this article.

\section{Highlights}

- Falls are common among wheelchair and scooter users with multiple sclerosis (MS). Previous research has found that approximately $75 \%$ of wheelchair and scooter users with MS report at least one fall in a 6-month period.

- Falls commonly occur while a wheelchair or scooter user is performing a transfer, reaching for an object, or ambulating short distances. Targeted education is necessary to enhance safety during these common activities and movements.

- Falls frequently occur in the wheelchair or scooter user's bathroom. Home assessments are critical to assess the interaction between physical abilities and exposure to environmental stressors. When possible, modifications should be made to enhance usability and safety.

- The diversity of fall attributions (i.e., intrinsic and extrinsic factors associated with falls) among people with MS who use wheelchairs or scooters suggests that opportunities to create and address individualized fall prevention goals during fall prevention education programs, is a priority.

- Focused research efforts, including large, prospective studies are necessary to further understand the factors influencing falls in people with MS who use wheelchairs and scooters.

- Additional intervention research is necessary to foster evidenced-based approaches to fall prevention for people with MS who use wheelchairs and scooters.

Multiple sclerosis (MS) is a chronic inflammatory demyelinating disease of the central nervous system and the most common non-traumatic cause of functional disability among young adults. Approximately 1 million people in the United States and 2.5 million worldwide live with $\mathrm{MS} .{ }^{2}$ Although it is presumed to be an autoimmune disease caused by a variety of factors, the precise etiology remains unknown. The disease process associated with MS often leads to muscle weakness, spasticity, and sensory disturbances, as well as impairments such as gait dysfunction and impaired postural control.' Approximately $25 \%$ of individuals living with MS are unable to ambulate functional distances in their home.,3 For these individuals, wheeled mobility devices, such as manual wheelchairs, power wheelchairs, or scooters, are important to support functional mobility. 
A recent study of 44 wheelchair and scooter users living with MS found that $75 \%$ of participants reported at least one fall in a 6-month period. ${ }^{5}$ The physical and psychological consequences of falls for persons with MS are increasingly well-documented ${ }^{5,6}$ and early evidence clearly suggests that fulltime wheelchair and scooter users are subjected to negative post-fall sequelae. Among the participants in the study described above, $76.7 \%(n=33 / 43)$ reported concerns about falling and $65.9 \%(n=29 / 44)$ limited their activities because of concerns. These findings, though based on a small sample, were surprisingly consistent with an earlier study that involved a cohort of 1,046 ambulatory and non-ambulatory individuals with MS.? In that study, $63.5 \%$ reported fear of falling. Among participants reporting fear of falling, $82.6 \%$ reported activity curtailment.? Activity curtailment associated with fear of falling is especially problematic due to its potential to contribute to physical deconditioning, increased fall risk, ${ }^{8,9}$ and potential negative impact on community-based activity. Community-based activity, which involves being active in family and community life and engaging in typical roles and responsibilities in society, ${ }_{10}$ is critically important to fulltime wheelchair users because it supports their health and well-being, ${ }^{11}$ and is strongly associated with quality of life. ${ }^{12}$ Among wheelchair users, being an active member of society is a key factor in preventing health deterioration. ${ }^{13}$

When a fall does occur, initial recovery, getting off the ground, and returning to a desired position, can be a challenge. Among older adults, long lie times (i.e., lying on the ground for longer than 1 hour) have been associated with serious injury, hospitalization, and a move to a long-term care facility. ${ }^{14} \mathrm{~A}$ study of ambulatory people with MS found that $27 \%$ of fallers lay on the ground for at least 10 minutes, and $4.6 \%$ experienced a long lie. ${ }^{15} \mathrm{~A}$ study of power wheelchair users with various disabilities, including MS, found that the majority of participants required assistance to recover from a fall and $15 \%$ lay on the ground for at least 10 minutes. ${ }^{16}$ While the research on post-fall experiences among wheelchair and scooter users with MS is not yet available, it is reasonable to consider that the documented consequences of a post-fall long lie may also be experienced by people with MS who are fulltime wheelchair or scooter users.

Due to the differences in physical characteristics (e.g., muscle strength and balance impairments) and functional mobility limitations (e.g., assistive devices utilized), risk factors for falls are most likely distinct for fulltime wheelchair or scooter users with MS compared to those who ambulate. Likewise, fall prevention interventions that are effective for ambulatory people with MS may not work on fulltime wheelchair or scooter users. Thus, in order to inform interventions that have the potential to effectively minimize the frequency and severity of falls among fulltime wheelchair or scooter users with MS, we have undertaken this review to address the following objectives: a) summarize findings regarding influences on falls among fulltime wheelchair and scooter users with MS, as well as associated clinical implications informed by the authors' clinical experiences and biomechanical principles; b) describe the status and key features of intervention research to date focused on fall prevention for wheelchair and scooter users with MS; and c) present priorities for future research.

\section{Factors associated with falls among wheelchair and scooter users with multiple sclerosis}

Among fulltime wheelchair and scooter users living with MS, two studies have examined factors associated with falls. ${ }^{5.6}$ An international survey-based study ${ }^{5}$ examined fall prevalence and factors associated with falls among 44 fulltime wheelchair and scooter users with MS with
Figure 1: Factors influencing falls among wheelchair and scooter users with multiple sclerosis

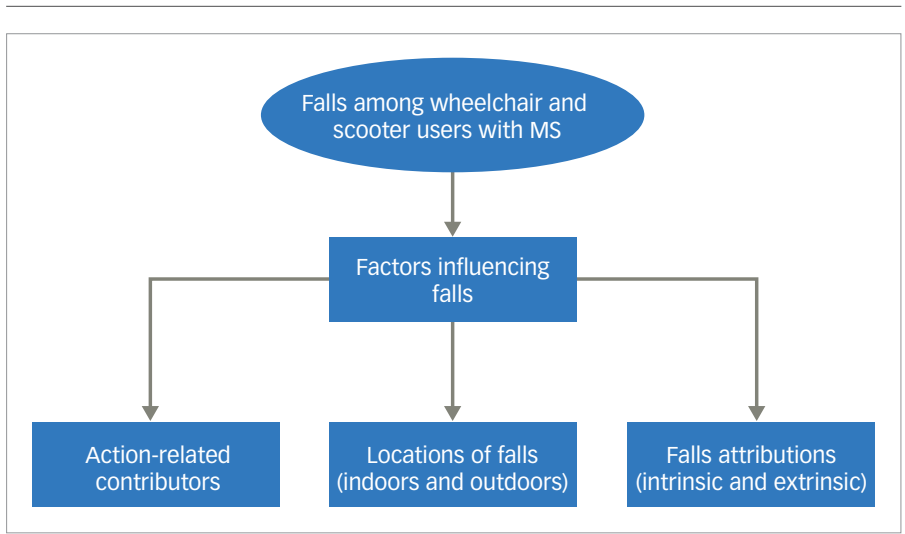

MS = multiple sclerosis.

Expanded Disability Status Scale ${ }^{17}$ (EDSS) scores between 7.0 and 7.5. This cross-sectional study collected data on the number of falls sustained by study participants, location of falls, activities being performed at the time of the fall, concerns about falling, and activity limitations associated with concerns about falling. The majority of participants in the study were female (74.4\%) with a mean age of $58.8 \pm 12.6$ years and used a mobility device an average of $35.6 \pm 19.3$ hours per week. The most common types of MS reported by participants was primary progressive $(n=19,43 \%$, previously reported as progressive/relapsing [27\%] and primary progressive [16\%]). Of the study participants, $75 \%(n=33)$ reported at least one fall in the previous 6 months. ${ }^{5}$

The second study analyzed the circumstances associated with a fall among wheelchair and scooter users with MS and spinal cord injury through a cross-sectional, mixed methods study. ${ }^{6}$ Participants self-reported the number of falls experienced in the past 6 months and completed surveys to examine fear of falling and associated activity curtailment. Next, participants described, in detail, the circumstances associated with their most recent fall in the past 6 months. The study included 18 wheelchair users with MS, of which 13 were female and had a mean age of $56.1 \pm 9.8$ years. Participants lived with MS for an average of $20.1 \pm 17.3$ years and used a wheelchair for an average of $5.3 \pm 6.1$ years. Half of the participants with MS used a manual wheelchair and half used a power wheelchair. ${ }^{6}$

To examine participants' descriptions of the most recent fall sustained, a thematic analysis was performed. ${ }^{18}$ To organize the findings, sung et al. ${ }^{6}$ proposed a classification system that includes three general categories: action-related fall contributors, the location of falls, and fall attributions (Figure 1). These categories will be used to report on findings from relevant studies, as well as clinical implications.

\section{Action-related fall contributors}

Results from both of the above studies indicate that transfers and reaching for objects are commonly associated with falls. ${ }^{5.6}$ Sung et al. found that transfers were associated with $55 \%$ of falls among fulltime wheelchair users. ${ }^{6}$ This is not surprising given the frequency of transfers fulltime wheelchair and scooter users perform, and the variety (e.g., wheelchair or scooter to/from a bed, car, toilet, tub). Rice et al. also showed that transfers (13.9\%) and activities that commonly involve transfers, such as taking a shower (11\%) and using the toilet (19.3\%), were frequently associated with 
falls among wheelchair or scooter users with MS. ${ }^{5}$ In addition, reaching for objects has also been found by Rice et al..$^{5}(13.9 \%)$ and Sung et al. ${ }^{6}(6 \%)$ to be associated with falls. Findings from both these studies highlight the risk of falling for fulltime wheelchair or scooter users with MS while ambulating short distances. Findings indicate that $14-33 \%$ of wheelchair and scooter users with MS reported falling while walking short distances despite using a wheelchair or scooter for the majority of their mobility. 5.6

\section{Clinical implications}

Transfer training, educating persons with MS about reaching beyond the base of support, and rehabilitation/habilitation efforts focusing on safety while ambulating short distances, may help to reduce fall risk among fulltime wheelchair and scooter users with MS. Transfer skill assessment and training must involve observed performance of transfers in a variety of situations, as opposed to reliance on a client's self-report. Using a validated assessment tool, such as the Transfer Assessment Instrument, ${ }^{19}$ may help to objectively evaluate the skills. Recognizing that people with MS may use different assistive devices for different needs, ${ }^{20}$ advice on the best options for assistive devices, and training in use of all prescribed assistive devices (e.g., standard or rolling walkers, quad canes) is warranted. Physical and occupational therapists can work together to build a client's functional mobility skills, ideally in the natural context.

Individuals with MS can be taught to proactively recognize situations that may result in reaching outside one's base of support and the associated fall risk, which involves not only displacing center of mass out of the base of support, but also the potential to displace the center of mass outside of the base of support when trying to regain control after an unexpected loss of balance. ${ }^{21,22}$ Safe reaching practices, from standing or wheelchair level, as well as compensatory strategies (e.g., moving commonly used items) can be incorporated into fall prevention education efforts and are ideally addressed in the context of functional activities (e.g., dressing, bathing).

Brief, but targeted, interviews undertaken with the goal of understanding when and where ambulatory episodes occur support a patient-centered approach to ambulation education and training. Discussions about home accessibility (described in detail later in this paper) may logically follow conversations about when ambulation occurs, since some people with MS may transition to fulltime wheelchair or scooter use before the home is made accessible. A home safety assessment may be warranted to ensure the wheelchair or scooter is compatible with the client's home and can be used in all critical areas. Creating practice opportunities that simulate or (preferably) replicate the situations that require ambulation appear to be vitally important to fall prevention programs. Finally, efforts to improve safety with transfers, reaching, and ambulating short distances may be enhanced by including caregivers in related education efforts. In a qualitative study examining falls self-efficacy among people with MS, "taking personal responsibility to reduce fall risk" emerged as a sub-theme..$^{25}$ The importance that study participants placed on leading their own fall-prevention efforts serves as a reminder to leave decisions regarding who will, or will not be involved in prevention efforts up to the client, when cognitive status supports such decision making. ${ }^{25}$

\section{Location of falls}

Available evidence indicates that among people with MS who are fulltime wheelchair or scooter users, indoor falls are more common than outdoor
Table 1: Location of falls reported by Sung et al. ${ }^{6}$

\begin{tabular}{|l|l|l|}
\hline Location & $\begin{array}{l}\text { Number of falls } \\
(n=12)\end{array}$ & $\%$ \\
\hline Indoor & \multicolumn{2}{|l|}{} \\
\hline Bathroom & 3 & 25 \\
\hline Bedroom & 2 & 17 \\
\hline Living room & 2 & 17 \\
\hline Kitchen & 1 & 8 \\
\hline Outdoor & 2 & 17 \\
\hline Garage & 2 & 17 \\
\hline Garden/yard & 2 & \\
\hline
\end{tabular}

falls. Rice et al. found that almost $90 \%$ of falls among wheelchair and scooters users occur inside of the home; however, the specific location of the fall was not reported. ${ }^{5}$ Among the falls examined by Sung et al., $25 \%$ occur in the bathroom, 17\% in the bedroom, 17\% in the living room, and $8 \%$ in the kitchen. Table 1 provides a breakdown of the fall locations described by sung et al. ${ }^{\circ}$

The high prevalence of indoor falls among wheelchair or scooter users with MS may be driven by the amount of time spent indoors and/or curtailment of activity outside the home. It is important to note that among fulltime wheelchair users, effective home modifications support greater participation in both home and community activities. ${ }^{24}$ Thus, unmet home modification needs could be contributing to both fall risks in the home and the amount of time spent indoors. Additionally, wheelchair and scooter users with MS perform very complex tasks (e.g., toileting, transferring to/from the shower) in the home, which may increase their risk of falling in distinct locations.

Regarding outdoor falls, Sung et al. found that $17 \%$ of falls occur in the garage, and 17\% occurred in the garden/yard, as described in Table $1 .{ }^{6}$ Transferring to or from a wheelchair/scooter to a car can be very challenging as a result of the gap between the device and car, and potentially extreme changes in height. An investigation that examined powered-wheelchair users with varied disabilities, reported falls on the street or sidewalk as the most common outside fall location. ${ }^{16}$ Falls on the street are highly influenced by environmental factors as well as by the intrinsic fall attributions. ${ }^{16}$

\section{Clinical implications}

Findings to date, regarding locations of falls, suggest a variety of clinical implications, ranging from assessment to intervention strategies. With respect to assessment priorities, healthcare providers can initiate conversations with clients to discover where they are spending their time and the activities occurring in those locations. Occupational therapists often perform assessments to determine how clients spend their time using validated instruments, such as the Occupational Questionnaire ${ }^{25}$ or the National Institutes of Health Activity Record. ${ }^{26}$ Clinicians can also ask follow-up questions to better understand the client's rationale for views regarding locations that are, or are not, safe and if the activities undertaken in those locations match personal ability levels.

A number of instruments are available to assess safety in and around the home including the Westmead Home Safety Assessment. ${ }^{27}$ Also, The Falls Prevention Home and Community Safety Online Workshop 
(wuw.fallspreventiononlineworkshops.com.au/) is available to train healthcare providers to conduct a comprehensive home visit to reduce the risk of falls.

Although developed for older community-residing adults, such resources can inform work with people with MS. Both the Westmead Home Safety Assessment and the aforementioned online workshop emphasize that home safety assessments involve more than simply determining the presence or absence of fall hazards in the home. Comprehensive assessments involve assessing interaction between the client's physical abilities and their exposure to environmental stressors. Given the finding that people with MS who are wheelchair or scooter users often experience outdoor falls occurring at or near the home ${ }_{1}{ }^{6}$ the importance of including these areas in home safety assessments cannot be overemphasized. Although the effectiveness of comprehensive home safety assessments for people with MS who are fulltime wheelchair or scooter users is not known, robust evidence from studies involving older adults indicates that home safety assessment and modification interventions are effective in reducing rate of falls and risk of falling..$^{28,29}$ Assessing community-based fall hazards can be challenging; however, tools such as the Community Health Environment Checklist, ${ }^{30}$ can be used to objectively measure the aspects of the physical environment that are important to persons with mobility impairments.

Creating opportunities for clients to practice valued activities in the locations where they typically occur to help the client accurately self-assess abilities and improve safety associated with performance, is clearly an intervention priority. The high prevalence of bathroom falls combined with the potential for injury, due to the (typically) small space and number of hard surfaces, means it is imperative for healthcare providers to build the client's capacity to safely engage in bathroom-based activities. Ideally, practice opportunities should occur in the client's home environment, but when this is not possible, clinicians can identify and create an environment which simulates the client's bathroom as closely as possible. Practicing strategies to manage fall risks in community-based settings is also important and has the potential to foster social integration and participation.

The findings by Rice et al. ${ }^{5}$ and Sung et al. ${ }^{6}$ highlight the importance of creating post-fall management plans that are not limited to management of indoor falls. Whether falls occur indoors or outdoors, caregivers may need to be involved in post-fall support to avoid delayed initial recovery or long lies. Clinicians can engage both the person with MS and caregivers to create fall recovery plans that support the safety of both.

\section{Fall attributions}

The circumstances in which falls occur among fulltime wheelchair and scooter users are multifactorial with various attributions contributing to the complexity. Previous studies show that these factors can be further classified as intrinsic or extrinsic factors. ${ }^{6,31}$ Intrinsic factors are related to the diagnosis of MS and the impact of fluctuating symptoms. ${ }^{31}$ Intrinsic risk factors are also related to individual actions, (e.g., distraction or forgetting to engage wheel locks). Extrinsic factors refer to factors related to the environment (e.g., cluttered environment, surface condition), which are closely related to the previously discussed location-related factors, as well as human interference, driving characteristics, or wheelchair malfunction (e.g., equipment failure). ${ }^{16,31}$
Sung et al. found that $43 \%$ of study participants with MS reported muscle weakness or spasticity as an intrinsic risk factor influencing falls, and $15 \%$ reported that loss of balance was responsible. ${ }^{6}$ It was also found that that $21 \%$ of wheelchair and scooter users with MS reported surface condition as an extrinsic factor influencing falls. ${ }^{6}$ It has also been shown, among powered-wheelchair users with various disabilities, including MS, that unfamiliar or inaccessible (e.g., steep inclines, poor lighting, cluttered environments) are common environmental risk factors. ${ }^{16}$

Like older adults, it appears that people with MS who are fulltime wheelchair or scooter users experience falls caused by diverse and interacting risk factors. For example, in a bathroom (location), transferring from a wheelchair to a toilet or tub (activity) can be very challenging for a person with MS who has leg weakness or balance impairments (fall attribution, intrinsic factor).

\section{Clinical implications}

The wide variation in intrinsic fall risk factors among people with MS makes careful assessment and targeted, individualized interventions a necessity. As potentially important contributors to falls, balance, leg weakness and spasticity warrant careful attention. Fortunately, evidence indicates that people with MS can improve balance and leg strength and reduce or manage spasticity through targeted interventions. ${ }^{32,33}$ Home exercise programs can be developed for these areas, with the client, in order to maximize adherence and long-term use. Efforts to address fall attributions must include attention to a variety of wheelchair and/or scooter use skills. These skills range from, managing challenging environments (e.g., slick surfaces, steep ramps, high curb cuts, crowds) to building safe habits (e.g., routinely remembering to engage wheel locks).

Wheelchair and/or scooter maintenance is a priority. It is important to provide education about resources such as local Centers for Independent Living and the National Multiple Sclerosis Society that can provide funding for new mobility devices or mobility device repair, and the importance of developing a strong working relationship with wheelchair vendors to assure the wheelchair or scooter continues to meet the individual's needs. Likewise, the rehabilitation team and/or assistive technology professionals can collaboratively ensure proper seating and positioning is achieved and maintained within the wheelchair or scooter seat.

In general, clinicians have important opportunities to initiate conversations about falls when a person with MS is seen after a fall-related injury, or presents with an MS symptom or situation that has the potential to increase fall risk. These conversations can focus on the diverse contributors to falls and their great potential for modification. Fear of disease progression and dependency are just two of the many reasons that a person with MS may be reluctant to bring up the topic of falls. Highlighting the interprofessional teams' expertise and eagerness to support the person with MS in his or her efforts to manage fall risks and safely participate in valued activities may help clients engage in honest conversations about this often-difficult subject.

\section{Evidence-based interventions to prevent falls in wheelchair users with multiple sclerosis}

The identification of factors influencing falls among people with MS lays the groundwork for the development and testing of targeted interventions designed to reduce fall incidence. ${ }^{34}$ Several interventions have been 
developed for ambulatory individuals with the purpose of preventing or reducing fall incidence. ${ }^{35-39}$ However, a significant limitation was found in the literature regarding falls prevention intervention studies specifically designed for fulltime wheelchair and scooter users with MS.

Only one study was found that reported the feasibility and preliminary results of an intervention focused on improving transfer skills and seated postural control in order to decrease fall frequency and concern about falling in fulltime wheelchair and scooters users with MS. ${ }^{40}$ Sixteen participants were enrolled with a mean age of 58 years, who had lived with MS for an average of 17 years. The majority of participants were female (69\%) and used a manual wheelchair (56.3\%). At the start of the intervention, participants reported an average of $1.7 \pm 2.7$ falls per month. The intervention program consisted of one 45-minute education session, in which individualized transfer education was provided by a trained physical therapist in a research laboratory setting. Participants were also provided with exercises to improve core muscle strength. After the education session, participants were given a manual detailing the transfer education and exercise program for review at home. Participants were asked to perform the exercise program three times per week and review the transfer education once a week at home. After 12 weeks of monitoring, fall frequency significantly decreased $\left(p<0.001, d_{z}=0.26\right)$, and transfer quality, ${ }_{1}^{41}$ as well as seated postural control, significantly improved $\left(p=0.01, d_{z}=0.97\right.$ and $p=0.02, d_{z}=0.83$, respectively). ${ }^{40}$ No significant differences were found regarding concerns about falling, as measured by the Spinal Cord Injury Fall Concerns Scale $\left(p=0.728, d_{z}=0.01\right)$. Qualitative results indicated that participants found the education helpful and changed the way transfers were performed. Although the low number of participants limits the generalizability of the findings, the intervention program was found feasible and positively reviewed by study participants. Preliminary results indicate that the education program was effective in decreasing fall frequency among wheelchair and scooter users with MS.

\section{Priorities for future research}

Although the amount of scientific interest concerning falls in wheelchair and scooter users with MS is growing, a focused research effort is needed to support the fall prevention needs of this population. Two studies were found that examined influences on fall risk among people with MS who are fulltime wheelchair or scooter users; however, prospective studies involving larger, diverse populations are necessary to determine fall risk factors in this population. Multicenter studies will most likely be needed to recruit the number of participants necessary for robust observational studies.

The need exists for evidence-based fall management programs that address physical, behavioral, attitudinal, and environmental fall risk factors appropriate for fulltime wheelchairs or scooters. Randomized trials are needed to assess the efficacy and effectiveness of such interventions. Given the wide range of actions, locations, and attributions that influence fall risk, programs that allow for development and attainment of individualized fall prevention goals appear to be a priority.
Pre-existing programs developed for wheelchair and scooter users may be valuable components of a fall prevention program; however, further evaluation is needed. For example, the Wheelchair Skills Training Program and Test is an excellent resource to both train and evaluate wheelchair skills. The validity of the program is well documented;42-44 however, the effect on wheelchair and scooter users with MS and potential to reduce falls is unclear. ${ }^{45}$ Over time, trial findings can help to determine the most effective timing for fall prevention programs developed for fulltime wheelchair or scooter users (e.g., when a client is a new versus long-time user), the optimal intervention duration needed to foster long-term benefit, and optimal intervention settings (e.g., home health, community-based programs).

Further research to understand and mitigate fear of falling among those with MS who are fulltime wheelchair or scooter users is needed. Importantly, the Falls Self Efficacy Scale-International, which is frequently used to measure fear of falling in older adults, ${ }_{16}{ }^{46}$ and validated for people with $\mathrm{MS}_{1}{ }^{47}$ cannot be used for fulltime wheelchair users because it includes ambulatory activities. Thus, the development and validation of an effective tool to measure fear of falling for people with MS who use wheeled mobility is needed.

Qualitative research is needed to further understand contextual details that yield realistic and useful fall prevention interventions for this population, as well as the fall prevention needs, preferred strategies, and the role of the caregiver for a person with MS who is a fulltime wheelchair user. In addition, qualitative work to understand when and how people with MS make decisions to move to wheeled mobility, and how these decisions may be related to fall risk, are indicated.

Finally, Finlayson et al. ${ }^{48}$ poignantly discusses the need to include participation as a long-term outcome in fall prevention intervention research for people with MS. The authors highlight that participation should be "about enabling and empowering individuals with MS to choose and engage in activities that are important and meaningful in their lives while simultaneously reducing risk of falls." ${ }^{\prime 8}$ The Community Participation Indicators ${ }^{49}$ or Impact on Participant and Autonomy Questionnaire ${ }^{50}$ are recommended measures of participation. ${ }^{48}$ Importantly, qualitative studies are needed to better understand the meaning and role of participation in the context of fall prevention efforts involving people with MS who use wheelchairs or scooters.

\section{Conclusion}

This review highlights the diverse influences on fall risk for fulltime wheelchair or scooter users with MS, as well as the need for well-designed studies to evaluate comprehensive fall prevention programs developed specifically for this population. In the absence of clear practice directives, clinicians are advised to utilize evidence-informed strategies as well as the expertise of the interprofessional team to best support fulltime wheelchair or scooter users with MS in their efforts to prevent falls. MS and fall prevention researchers have a number of unique opportunities to make important contributions to the development of fall prevention resources for this vulnerable population. $\square$
1. Noseworthy $\mathrm{JH}$, Lucchinetti C, Rodriguez $\mathrm{M}$, Weinshenker BG. Multiple sclerosis. N Eng/ J Med. 2000;343:938-52.

2. National Multiple Sclerosis Society. Who gets MS? www. nationalmssociety.org/What-is-MS/Who-Gets-MS (accessed March 16, 2018).

3. Chruzander C, Johansson S, Peterson EW, et al. Falls among non-ambulatory individuals with multiple sclerosis: an international expert panel consensus statement. Presented at: 2014 Joint ACTRIMS-ECTRIMS Meeting, Boston, MA, US September 10-13, 2014. Abstr. \#64532.

4. Einarsson U, Gottberg K, Fredrikson S, et al. Multiple sclerosis in Stockholm County. A pilot study exploring the feasibility of assessment of impairment disability and handicap by home visits. Clin Rehabil. 2003;17:294-303.

5. Rice L, Kalron A, Berkowitz SH, et al. Fall prevalence in people with multiple sclerosis who use wheelchairs and scooters. Medicine (Baltimore). 2017;96:e7860.

6. Sung J, Trace Y, Peterson EW, et al. Falls among fulltime wheeled 
mobility device users with spinal cord injury and multiple sclerosis: A comparison of characteristics of fallers and circumstances of falls. Disabil and Rehabil. 2017:25:1-7.

7. Peterson $\mathrm{EW}$, Cho $\mathrm{CC}$, Finlayson ML. Fear of falling and associated activity curtailment among middle aged and older adults with multiple sclerosis. Mult Scler. 2007;13: 1168-75.

8. Friedman SM, Munoz B, West SK, et al. Falls and fear of falling: which comes first? A longitudinal prediction model suggests strategies for primary and secondary prevention. J Am Geriatr SOC. 2002:50:1329-35.

9. Delbaere K, Crombez G, Vanderstraeten G, et al. Fear-related avoidance of activities, falls and physical frailty. A prospective community-based cohort study. Age Ageing. 2004;33:368-73

10. Rosenbaum P, Stewart D. The World Health Organization International Classification of Functioning, Disability, and Health: a model to guide clinical thinking, practice and research in the field of cerebral palsy. Semin Pediatr Neurol. 2004;11:5-10.

11. Liptak GS. Health and well being of adults with cerebral palsy. Curr Opin Neurol. 2008;21:136-42.

12. Ravenek KE, Ravenek MJ, Hitzig SL, Wolfe DL. Assessing quality of life in relation to physical activity participation in persons with spinal cord injury: a systematic review. Disabil Health 2012;5:213-23.

13. Integration into the Community. In: Trieschmann RB. Spinal Cord Injuries: Psychological, Social, and Vocational Rehabilitation. 2nd ed. New York: Springer Publishing Company, 1988;147-236.

14. Fleming J, Brayne C, Cambridge City over-75s Cohort (CC75C) study collaboration. Inability to get up after falling, subsequent time on floor, and summoning help: prospective cohort study in people over 90. BMJ. 2008;337:a2227.

15. Bisson EJ, Peterson EW, Finlayson M. Delayed initial recovery and Iong lie after a fall among middle-aged and older people with multiple sclerosis. Arch Phys Med Rehabil. 2015;96:1499-505.

16. Rice LA, Sung J, Peters J, et al. Perceptions of fall circumstances, injuries and recovery techniques among power wheelchair users: a qualitative study. Clin Rehabil. 2018;32:985-93.

17. Kurtzke JF. Rating neurologic impairment in multiple sclerosis: an expanded disability status scale (EDSS). Neurology. 1983;33:1444-52.

18. Braun V, Clarke V. Using thematic analysis in psychology. Qual Res Psychol. 2006;3:77-101.

19. Worobey LA, Zigler CK, Huzinec $R$, et al. Reliability and validity of the revised Transfer Assessment Instrument. Top Spinal Cord Inj Rehabil. 2018:24:217-26.

20. Finlayson ML, Peterson EW, Asano M. A cross-sectional study examining multiple mobility device use and fall status among middle-aged and older adults with multiple sclerosis. Disabil Rehabil Assist Technol. 2014;9:12-6.

21. Larson CA, Tezak WD, Malley MS, Thornton W. Assessment of postural muscle strength in sitting: reliability of measures obtained with hand-held dynamometry in individuals with spinal cord injury. I Neurol Phys Ther. 2010:34:24-31.

22. Jorgensen V, Elfving B, Opheim A. Assessment of unsupported sitting in patients with spinal cord injury. Spinal Cord. 2011;49:838-43.

23. Peterson E, Kielhofner $G$, Tham $K$, van Koch L. Falls self-efficacy among adults with MS: A phenomenological study. OTJR. 2010;30:148-57.

24. Harris $F$, Yang $H$, Sanford J. The impact of home modifications and wheelchair usability on activity and participation. Presented at: Rehabilitation Engineering and Assistive Technology Society of North America (RESNA) Annual Conference, Toronto, Canada, June $5-8,2011$

25. Smith NR, Kielhofner G, Watts JH. The relationships between volition, activity pattern, and life satisfaction in the elderly. Am J occup Ther. 1986;40:278-83.

26. Furst GP, Gerber LH, Smith CC, et al. A program for improving energy conservation behaviors in adults with rheumatoid arthritis. Am J Occup Ther. 1987:41:102-11.

27. Clemson L, Fitzgerald MH, Heard R. Content validity of an assessment tool to identify home fall hazards: The Westmead Home Safety Assessment. Br J Occup Ther. 1999;62:171-9.

28. Gillespie LD, Robertson MC, Gillespie WJ, et al. Interventions for preventing falls in older people living in the community. Cochrane Database Syst Rev. 2012:CD007146.

29. Hopewell S, Adedire O, Copsey BJ, et al. Multifactorial and multiple component interventions for preventing falls in older people living in the community. Cochrane Database Syst Rev. 2018;7:CD012221

30. Stark S, Hollingsworth HH, Morgan KA, Gray DB. Development of a measure of receptivity of the physical environment. Disabil Rehabil. 2007;29:123-37.

31. Carling A, Forsberg A, Nilsagard Y. Falls in people with multiple sclerosis: experiences of 115 fall situations. Clin Rehabil. 2018;32:526-35.

32. Sosnoff J, Motl RW, Snook EM, Wynn D. Effect of a 4-week period of unloaded leg cycling exercise on spasticity in multiple sclerosis. NeuroRehabilitation. 2009;24:327-31.

33. Sosnoff JJ, Motl RW. Effect of acute unloaded arm versus leg cycling exercise on the soleus $\mathrm{H}$-reflex in adults with multiple sclerosis. Neurosci Lett. 2010;479:307-11.

34. Cameron M, Coote S, Sosnoff JJ. Whom to target for falls-prevention trials: recommendations from the Internationa MS Falls Prevention Research Network. Int I MS Care. 2014;16:203-7.

35. Coote S, Hogan N, Franklin S. Falls in people with multiple sclerosis who use a walking aid: prevalence, factors, and effect of strength and balance interventions. Arch Phys Med Rehabil. 2013;94:616-21.
36. Sosnoff JJ, Finlayson M, McAuley E, et al. Home-based exercise program and fall-risk reduction in older adults with multiple sclerosis: phase 1 randomized controlled trial. Clin Rehabil. 2014:28:254-63.

37. Finlayson $M$, Peterson EW, Cho C. Pilot study of a fall risk management program for middle aged and older adults with MS. NeuroRehabilitation. 2009;25:107-15

38. Cattaneo D, Jonsdottir J, Zocchi M, Regola A. Effects of balance exercises on people with multiple sclerosis: a pilot study. Clin Rehabil. 2007;21:771-81.

39. Hugos CL, Frankel D, Tompkins SA, Cameron M. Community delivery of a comprehensive fall-prevention program in people with multiple sclerosis: a retrospective observational study. Int MS Care. 2016;18:42-8.

40. Rice LA, Isaacs Z, Ousley C, Sosnoff J. Investigation of the feasibility of an intervention to manage fall risk in wheeled mobility device users with multiple sclerosis. Int I MS Care. 2018;20:121-8.

41. Tsai $C Y$, Rice LA, Hoelmer C, Boninger ML, Koontz AM. Basic psychometric properties of the transfer assessment instrument (version 3.0). Arch Phys Med Rehabil. 2013;94:2456-64.

42. Best KL, Kirby RL, Smith C, MacLeod DA. Wheelchair skills training for community-based manual wheelchair users: a randomized controlled trial. Arch Phys Med Rehabil. 2005;86:2316-23.

43. Best KL, Miller WC, Huston G, et al. Pilot study of a peer-led wheelchair training program to improve self-efficacy using a manual wheelchair: a randomized controlled trial. Arch Phys Med Rehabil. 2016;97:37-44.

44. MacPhee AH, Kirby RL, Coolen AL, et al. Wheelchair skills training program: A randomized clinical trial of wheelchair users undergoing initial rehabilitation. Arch Phys Med Rehabil. 2004;85:41-50.

45. Tu CJ, Liu L, Wang W, et al. Effectiveness and safety of wheelchair skills training program in improving the wheelchair skills capacity: a systematic review. Clin Rehabil. 2017:31:1573-82.

46. Yardley L, Beyer N, Hauer K, et al. Development and initial validation of the Falls Efficacy Scale-International (FES-I). Age Ageing. 2005;34:614-9.

47. van Vliet $R$, Hoang P, Lord S, et al. Falls efficacy scale-international: a cross-sectional validation in people with multiple sclerosis. Arch Phys Med Rehabil. 2013;94:883-9.

48. Finlayson M, Peterson E, Matsuda PN. Participation as an outcome in multiple sclerosis falls-prevention research: consensus recommendation from the International MS Falls Prevention Research Network. Int J MS Care. 2014:16:171-7.

49. Heinemann AW, Lai JS, Magasi S, et al. Measuring participation enfranchisement. Arch Phys Med Rehabil. 2011;92:564-71.

50. Cardol M, de Haan RJ, van den Bos GA, et al. The development of a handicap assessment questionnaire: the Impact on Participation and Autonomy (IPA). Clin Rehabil. 1999;13:411-9. 